
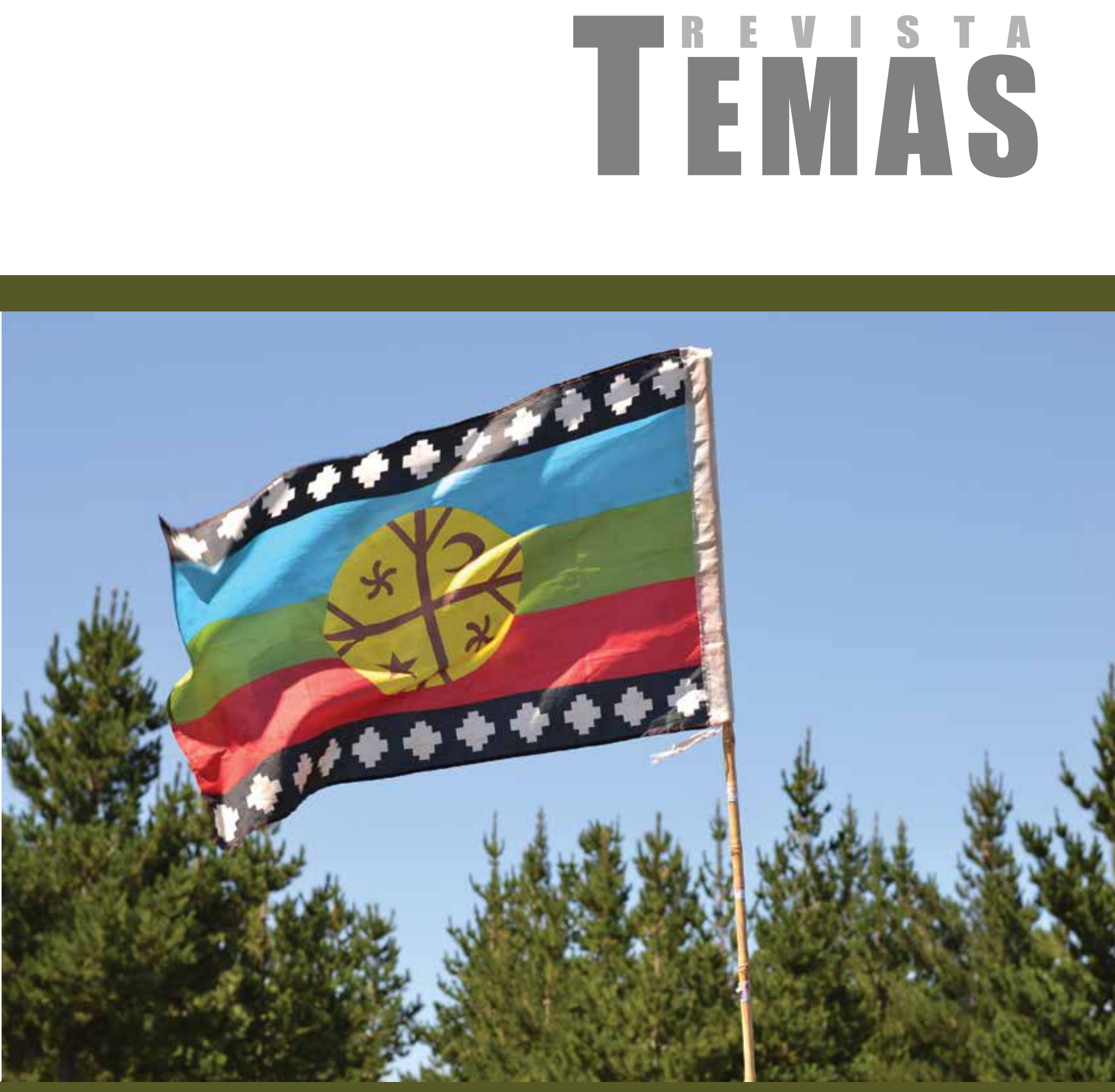

Bandera Mapuche. Chile, Enero del 2016. 

Referencia al citar este artículo:

Amaya, L. P., Rivera, R., y Acosta, H. U. (2020). Análisis de la relación entre el resultado de lectura crítica en las pruebas saber 11, el promedio académico y el desempeño en tareas de atención y memoria en estudiantes universitarios. Revista TEMAS, 3(10), 99 - 108.

\section{Análisis de la relación entre el resultado de lectura crítica en las pruebas Saber 11, el promedio académico y el desempeño en tareas de atención y memoria en estudiantes universitarios ${ }^{1}$}

Laura Patricia Amaya Díaz ${ }^{2}$ Raquel Rivera Carvajal ${ }^{3}$

Hadder Uriel Acosta Salazar

Recibido: 14 de abril de 2020 Aprobado: 6 de mayo de 2020

\section{Resumen}

Se analizó la relación entre el resultado de lectura crítica en las pruebas Saber 11, el rendimiento académico y el desempeño en tareas de atención y memoria en 55 estudiantes universitarios (edad media $=20$ años, D.T. $=2.51$ ) a través de la prueba Memometric, la subprueba de retención de dígitos de Wechsler y el test de cancelación de la A. Como resultado, se encontró que el rendimiento en el módulo de lectura crítica y el desempeño académico no se relacionan únicamente con procesos psicológicos básicos como la atención selectiva y la memoria de trabajo. Adicionalmente, las correlaciones entre las variables estudiadas fueron bajas (con puntuaciones entre $r=.004$ y $r=.42 ; p<.05$ ), a excepción de dos, que pueden ser consideradas como moderadas: inverso memometric y promedio académico con un $r, 50 \mathrm{p}<.05$; y directo memometric e inverso memometric, con un $r=, 67, p<.05$. Con el análisis posterior del modelo de regresión se observó que el desempeño en la prueba de lectura crítica Saber 11 fue significativo para la predicción del rendimiento académico. En conclusión, solo fueron corroboradas dos de las relaciones planteadas. Se sugiere para futuras investigaciones incluir variables relacionadas con los recursos afectivos del estudiante y los ambientes de aprendizaje.

\section{Palabras clave}

Memometric, memoria de trabajo, atención selectiva, rendimiento académico, pruebas de Estado, lectura crítica.

\section{Analysis of the Relationship between critical reading outcomes in the high school state test, academic grade point average (GPA) and performance in attention and memory tasks in university students}

1. Artículo de investigación científica y tecnológica.

2. Magíster en Educación por la Universidad Internacional de la Rioja, docente investigadora Universidad Manuela Beltrán Bucaramanga. Correo electrónico Lauraamaya3@hotmail.com

3. Magíster en epidemiología por la Universidad Industrial de Santander, docente investigadora Universidad de Santander UDES. Correo electrónico: raquelrivera_c@hotmail.com

4. Especialista en Necesidades Educativas e Inclusión por la Universidad Autónoma de Bucaramanga, docente Corporación Universitaria Minuto de Dios - UNIMINUTO. Correo electrónico: hadder.acosta@uniminuto.edu.co 


\section{Abstract}

In this study was analyzed the relation between the result of critical reading in the tests knowing 11 , the academic performance and the performance in tasks of attention and memory in 55 university students (average age $=20$ years, SD $=$ 2.51) through the memometric test, the Wechsler digit retention subtest and the A cancellation test. As a result, it was found that the performance in the critical reading module and academic performance are not related only to basic psychological processes such as selective attention and memory of work. Additionally, the correlations between the variables studied were low (with scores between $r=.004$ and $r=.42$; $p<.05$ ), except for two, which can be considered as moderate: inverse memometric and academic average with $r, 50 \mathrm{p}<.05$; and direct memometric and inverse memometric, with $r=, 67$, $p<.05$. With the subsequent analysis of the regression model, it was observed that the performance of the critical reading test knowing 11 was significant for the prediction of academic performance. In conclusion, only two of the proposed relationships were corroborated. It is suggested for future research to include variables related to the affective resources of the student and the learning environments.

\section{Keywords}

Memometric, working memory, slective attention, academic performance, Secondary school state test, critical reading.

\section{Introducción}

En Colombia, el Ministerio de Educación Nacional determina como requisito fundamental para acceder a la educación superior, además de obtener el título como bachiller, el haber presentado la prueba de Estado que realiza el Instituto Colombiano para la Evaluación de la Educación (ICFES) o su equivalente en otros países (Mineducación, 2016). En coherencia, este examen hace parte de los instrumentos estandarizados que miden la calidad del servicio educativo, suministrando información y elementos de referencia para la construcción de políticas educativas locales y nacionales (Decretos 869 de marzo de 2010; Decreto 3963 de octubre de 2009, MEN).

El examen de Estado Saber 11 está compuesto por 5 pruebas: lectura crítica, matemáticas, sociales y ciudadanas, ciencias naturales e inglés. La primera evalúa tres competencias que involucran habilidades cognitivas fundamentales para comprender el significado de las palabras y frases, establecer la relación de los componentes de una oración o enunciado, determinar la relación semántica y formal que se da entre diferentes elementos de un texto para alcanzar un sentido global (MEN, Decreto 3963 de 14 octubre de 2009; MEN, Decreto 869 de 17 marzo de
2010). Al respecto, cabe anotar que el puntaje en lectura crítica es un requisito fundamental para la admisión de estudiantes en las carreras que pertenecen a las ciencias sociales y humanas, por la relación del ejercicio profesional con procesos de comprensión, análisis, interpretación y argumentación.

González y Vega (2010) a partir de un estudio realizado sobre prácticas de lectura y escritura en cinco asignaturas de diferentes programas de la Universidad Sergio Arboleda, halló algunos datos de relevancia que evidencian la débil formación de los bachilleres, destacando que el $73,23 \%$ de los estudiantes que ingresan a la universidad manejan un tipo de lectura literal y un proceso de escritura básico, el $22,81 \%$ alcanza un nivel de lectura inferencial que permite la comprensión global de un texto, y tan solo un $3,94 \%$ de los estudiantes presenta un nivel de lectura y escritura avanzado. Lotti, Salim, Raya y Dori (2008) demuestran que las dificultades en procesos de comprensión y producción de textos influyen notablemente en el rendimiento de los estudiantes universitarios.

De acuerdo con Cáceres y Salas (2017) el rendimiento académico es un concepto pluridimensional, multicausal e incluso relativo y contextual. Muelas y Beltrán (2011) 
lo definen como un índice del progreso de los estudiantes en la adquisición de conocimientos y habilidades durante su formación profesional. A nivel general, se relaciona con estatutos simbólicos dentro de una escala numérica, producto de ejercicios de evaluación de los conocimientos adquiridos en el sistema educativo. A partir del desarrollo de los cursos de cada plan de estudios, se trabajan de forma transversal las competencias que las pruebas de estado miden; atendiendo a una estrategia educativa general. Diferentes investigaciones (Chamorro-Premuzic \& Furnham, 2008; Chamorro-Premuzic, Quiroga \& Colom, 2009; Colom \& Flores-Mendoza, 2007; Deary, Strand, Smith \& Fernández, 2007) se han centrado en determinar predictores de rendimiento académico, resaltando variables, como la inteligencia, la motivación, la memoria, la atención y los recursos afectivos.

Según Prada, Pineda, y Conde y Orduz (2010) se puede estimar que el desempeño en test atencionales como el de la cancelación de la $A$, se convierte en predictor de rendimiento en pruebas computarizadas que evalúan la memoria (Memonum). Lo anterior, argumentado en la relación entre la atención selectiva y la inhibición de estímulos irrelevantes. De acuerdo con Baddeley (1992)6a) la memoria de trabajo establece un vínculo fundamental entre la percepción, la atención y la acción.

El concepto de atención ha tenido múltiples transformaciones, desde el reconocimiento como filtro encargado de seleccionar la información hasta un banco de recursos que permite el procesamiento de distintas tareas. Investigaciones más recientes han permitido integrar dichas concepciones y considerar la atención como un conjunto de redes neurales responsables de tareas específicas del procesamiento de la información (Colmenero, Catena y Fuentes, 2001). Una de las funciones elementales consiste en la selección de estímulos sensoriales, producto de la información que llega a los sentidos. El proceso de atención se puede subdividir en varias fases, las cuales suponen dirigir, mantener, desatender y reorientar el foco a un lugar o punto determinado, diferenciando dos sistemas complementarios de la atención; uno automático y otro voluntario (Fernández, 2008).

El sistema voluntario de atención está asociado a las áreas dorsales del lóbulo frontal y parietal; funcional y anatómicamente relacionado con la capacidad de mantener información en la memoria. Por su parte, el sistema automático de atención se encuentra localizado en áreas ventrales del hemisferio derecho y funciona como una alerta general que indica la necesidad de reubicar la atención sin precisar a dónde va dirigida en el espacio visual (Fernández, 2008). La atención involucra diferentes tipologías (Cooley y Morris, 1990; Posner y Dehaene, 1994; Posner y Petersen, 1990; Stuss, 1995), una de ellas es la atención selectiva, responsable de seleccionar y controlar la información para que el individuo se desarrolle y responda a las demandas del medio.

La atención selectiva involucra dos fases que pueden presentarse de forma simultánea, la inicial, correspondiente a la selección espacial y, la segunda, fundamentada en las características del objeto (Vázquez, Vaquero, Cardoso y Gómez, 2001). La coexistencia de estas dos fases implica una adecuada coordinación binocular, permitiendo a través de las vías magnocelular y parvocelular procesar imágenes sin error (Álvarez et al. 2007).

Respecto a la conceptualización de la memoria, teóricos actuales la definen como una serie de sistemas complejos interconectados que tienen distintos propósitos y no como una simple función unitaria 
(Puente, 2010). Acorde con lo anterior, las distinciones más comunes son la memoria sensorial, la memoria a corto plazo y la memoria a largo plazo.

A partir de la clasificación descrita anteriormente, el presente estudio abordó el componente de la memoria a corto plazo, desde el planteamiento de memoria de trabajo de Baddeley (1992) quien la describe como un sistema de capacidad limitada y carácter temporal, que permite mantener y manipular información de manera simultánea en el desarrollo de tareas cognitivas complejas.

En este orden de ideas, la memoria de trabajo está integrada por varios componentes: el controlador central relacionado con la organización de los recursos en los procesos de codificación, almacenamiento de información y participación en acciones voluntarias, considerado el más complejo por la asociación con el funcionamiento de los lóbulos frontales.

Por otra parte, está el bucle articulatorio o fonológico, el cual se asocia a las funciones de almacenar la huella de la palabra en la memoria y el registro de nueva información. Baddeley adicionó un nuevo componente llamado buffer episódico, el cual es descrito como un sistema de almacenamiento simultáneo de datos que provienen del bucle fonológico y la agenda visoespacial, la cual al ser integrada con información guardada en el sistema de largo plazo crea una representación multimodal y temporal de la situación actual (Tirapu, García, Ríos \& Pelegrín, 2011).

El Grupo System-Bio basado en la prueba "Memonum" (Albarracín, Dallos, \& Conde, 2008) y la prueba de retención de dígitos de Wechsler (1945) diseñó el instrumento computarizado Memometric, el cual evalúa principalmente aspectos de la memoria de trabajo y permite simultáneamente manipular los intervalos de exposición, incluir cambios de colores como estímulos distractores y registrar variables relacionadas con aciertos y tiempos de respuesta.

Teniendo en cuenta las exigencias cognitivas en el proceso de formación de los profesionales en ciencias sociales, específicamente, por la adquisición y desarrollo de competencias relacionadas con lectura crítica y procesos psicológicos como la atención y la memoria, se formuló la pregunta: ¿Cómo se relacionan los resultados de los exámenes de Estado Saber 11, el rendimiento académico y el desempeño en tareas cognitivas de atención y memoria en estudiantes universitarios de la ciudad de Bucaramanga?

\section{Metodología}

\section{Tipo de estudio}

Se llevó a cabo un estudio no experimental, correlacional de corte transversal.

\section{Participantes}

Participaron de manera voluntaria, consentida e informada 55 estudiantes universitarios, con una media de edad de 20 años (D.T. $=2.51$ ) y un rango entre 17 y 26 años de sexo femenino y masculino, pertenecientes a la Facultad de Ciencias Sociales.

\section{Materiales y procedimiento}

Los participantes fueron evaluados individualmente en una sesión de aproximadamente 30 minutos.

Prueba Memometric: Basada en la prueba "Memonum" (Albarracín, Dallos, \& Conde, 2008) y la prueba de retención de dígitos de Wechsler (1945) evalúa principalmente aspectos de la memoria 
operacional. La prueba computarizada permite personalizar los tiempos de exposición y transición de los dígitos presentados, así como la inclusión de cambios de colores como estímulos distractores o de interferencia para observar su influencia sobre el desempeño mnemónico y atencional. Para el desarrollo de las pruebas realizadas se utilizó la versión de evocación en orden directo e inverso, sin interferencia distractora y con tiempo de exposición de dígitos de 2 segundos. En total, se presentaron 8 reactivos cada uno con dos ensayos. El máximo puntaje que se puede obtener en orden directo es 88 y en orden inverso 74 .

Test de Cancelación de la "A': Fue concebido como una medida de vigilancia y registro visual que valora la atención selectiva y sostenida (Ardila, Rosselli \& Puente, 1994; Estévez et al., 1997; Lezak, 1995). El participante debe cancelar todas las letras " $A$ " que encuentra en una planilla con 160 letras. Fueron calificados los aciertos (máximo 16). Adicionalmente, se registró el tiempo total de ejecución. Este instrumento se empleó para obtener un registro de los procesos atencionales.

Subprueba Retención de dígitos -Escala de inteligencia Wechsler para adultos- IV (WAIS-IV, 2012): Se aplicaron dos tareas: dígitos directos (consiste en repetir una serie de dígitos, que se presentan oralmente, en el mismo orden que se verbalizan) y dígitos inversos (repetir una serie de dígitos en orden inverso al presentado). Evalúa atención, resistencia a la distracción, memoria auditiva inmediata y la memoria de trabajo.

Resultados rendimiento académico del periodo cursado: Para los efectos de esta investigación, las categorías de bajo y alto desempeño se infirieron a partir de lo establecido en el reglamento académico de la institución donde se llevó a cabo el proyecto. En una escala de 1.0 a 5.0 se consideraron los puntajes obtenidos menor a 3.5 como bajo rendimiento y los superiores a 4.0 se catalogaron como alto rendimiento.

\begin{tabular}{|c|c|c|}
\hline \multicolumn{3}{|c|}{ RETENCIÓN DE DÍGITOS DIRECTO } \\
\hline REACTIVO & ENSAYO & $\begin{array}{c}\text { DÍGITOS } \\
\text { PRESENTADOS }\end{array}$ \\
\hline \multirow{2}{*}{1} & 1 & 2 \\
\hline & 2 & 2 \\
\hline \multirow{2}{*}{2} & 1 & 3 \\
\hline & 2 & 3 \\
\hline \multirow{2}{*}{3} & 1 & 4 \\
\hline & 2 & 4 \\
\hline \multirow{2}{*}{4} & 1 & 5 \\
\hline & 2 & 5 \\
\hline \multirow{2}{*}{5} & 1 & 6 \\
\hline & 2 & 6 \\
\hline \multirow{2}{*}{6} & 1 & 7 \\
\hline & 2 & 7 \\
\hline \multirow{2}{*}{7} & 1 & 8 \\
\hline & 2 & 8 \\
\hline \multirow{2}{*}{8} & 1 & 9 \\
\hline & 2 & 9 \\
\hline \multicolumn{3}{|c|}{ RETENCIÓN DE DÍGITOS INVERSO } \\
\hline REACTIVO & ENSAYO & $\begin{array}{c}\text { DÍGITOS } \\
\text { PRESENTADOS }\end{array}$ \\
\hline \multirow{2}{*}{1} & 1 & 2 \\
\hline & 2 & 2 \\
\hline \multirow{2}{*}{2} & 1 & 2 \\
\hline & 2 & 2 \\
\hline \multirow{2}{*}{3} & 1 & 3 \\
\hline & 2 & 3 \\
\hline \multirow{2}{*}{4} & 1 & 4 \\
\hline & 2 & 4 \\
\hline \multirow{2}{*}{5} & 1 & 5 \\
\hline & 2 & 5 \\
\hline \multirow{2}{*}{6} & 1 & 6 \\
\hline & 2 & 6 \\
\hline \multirow{2}{*}{7} & 1 & 7 \\
\hline & 2 & 7 \\
\hline \multirow{2}{*}{8} & 1 & 8 \\
\hline & 2 & 8 \\
\hline
\end{tabular}




\section{Procedimiento}

Como primera medida se contactó y se solicitó la autorización a la dirección académica para presentar el proyecto a los estudiantes. Una vez obtenido su consentimiento para participar, se procedió a la aplicación colectiva de los instrumentos, cuya duración fue de 30 minutos aproximadamente. La aplicación se realizó en las aulas de informática, previo acuerdo con los docentes.

\section{Resultados}

Para el análisis de datos se utilizó el programa SPSS versión 24.0. Según el nivel de significancia de las pruebas estadísticas, se organizaron en las categorías $\mathrm{P}<0,001, \mathrm{P}<0,01$ y $\mathrm{P}<0,05$. Los resultados que se presentan a continuación muestran, en primer lugar, los estadísticos descriptivos de las puntuaciones obtenidas por los participantes en los instrumentos aplicados $\mathrm{y}$, posteriormente, las correlaciones halladas entre las variables.
La muestra evaluada obtuvo un desempeño promedio correspondiente a $3.91 \pm$ 0.36 en rendimiento académico, $56.21 \pm$ 8.33 en la prueba de lectura crítica Saber 11. En las tareas de atención y memoria, las medianas y rangos intercuartílicos correspondieron a: total memometric en orden directo $\mathrm{Me}=64(52-70)$, total memometric en orden inverso $\mathrm{Me}=50$ $(39-56)$, total retención de dígitos directo prueba Wechsler $\mathrm{Me}=8.23 \pm 2.00 \mathrm{y}$ total retención de dígitos orden inverso prueba Wechsler Me= $6.67 \pm 1.93$ (tabla 1).

Al comparar el desempeño en rendimientos académico, se halló que los puntajes menores a 3.5 representaron el $12.73 \%$ (7), los de 3.5 a 4.0 el $47.27 \%$ (26) y los mayores a 4.0 el $40 \%$ (22). Se identificaron diferencias significativas en el desempeño en tares de atención y memoria específicamente en total memometric directo, total memometric inverso y total retención de dígitos inverso prueba Wechsler, con valores $\mathrm{p}$ según la prueba Kruskal Wallis $<0.05$.

Tabla 1. Análisis niveles de rendimiento académico

\begin{tabular}{|c|c|c|c|c|c|}
\hline \multirow{2}{*}{ Variable } & \multirow{2}{*}{$\begin{array}{c}\text { Total } \\
(n=55)\end{array}$} & \multicolumn{4}{|c|}{ Promedio académico } \\
\hline & & $<3.5(n=7)$ & $3.5-4.0(26)$ & $>4.0(n=22)$ & $\mathbf{P} *$ \\
\hline \multicolumn{6}{|l|}{ Sexo } \\
\hline Femenino & $74.55(41)$ & $85.71(6)$ & $73.08(19)$ & $72.73(16)$ & 0.834 \\
\hline Masculino & $25.45(14)$ & $14.29(1)$ & $26.92(7)$ & $27.27(6)$ & \\
\hline Edad & $20.29 \pm 2.51$ & $18(17-21)$ & $19.5(18-22)$ & $21(19-22)$ & 0.072 \\
\hline Lectura crítica Saber 11 & $56.21 \pm 8.33$ & $51.57 \pm 10.9$ & $55.76 \pm 7.44$ & $58.22 \pm 8.27$ & 0.175 \\
\hline \multicolumn{6}{|l|}{ Pruebas Memometric } \\
\hline Total Orden directo & $64(52-70)$ & $50(30-54)$ & $62(54-68)$ & $68(63-73)$ & 0.0019 \\
\hline \multirow[t]{2}{*}{ Total orden inverso } & $50(39-56)$ & $26(19-51)$ & $47(37-50)$ & $55.5(51-61)$ & 0.0003 \\
\hline & $33(27-47)$ & $32(27-40)$ & $38(30-52)$ & $30(25-42)$ & 0.0981 \\
\hline \multicolumn{6}{|l|}{$\begin{array}{l}\text { Subprueba retención de } \\
\text { dígitos Wechsler }\end{array}$} \\
\hline Total directo & $8.23 \pm 2.00$ & $9(6-9)$ & $8(7-9)$ & $5(7-9)$ & 0.9732 \\
\hline Total inverso & $6.67 \pm 1.93$ & $6(4-9)$ & $6(5-7)$ & $7(6-8)$ & 0.0445 \\
\hline
\end{tabular}




\section{Análisis de correlación}

Fue empleado el coeficiente de Spearman con la finalidad de estimar la relación de variables del desempeño en la prueba Memometric, retención de dígitos de Wechsler, test de cancelación de la A, resultados en la prueba de lectura crítica - Saber 11 y rendimiento académico. Se identificó que el promedio académico presenta correlación positiva con valores $p<0.0001$ con total memometric directo e inverso, con la subprueba de retención de dígitos Wechsler de manera positiva $(p<0.01)$ y con el tiempo de reacción en el test de cancelación de la $\mathrm{A}$ de manera negativa (figura1). Los resultados en la prueba de lectura crítica Saber 11 presentan correlación con la subprueba de retención de dígitos total inverso de Wechsler con un valor $p<0.01$.

\section{Análisis multiple}

El análisis múltiple se realizó mediante regresión lineal, al respecto se encontró que por cada unidad de aumento en el puntaje de la prueba total memometric inverso, los estudiantes presentan un aumento en el promedio académico de 0.0111 (0.0058; 0.0163) ajustado por las variables incluidas en el modelo, lo cual fue significativo también en el análisis crudo (tabla 2). En relación con el tiempo de reacción, por cada minuto que demoran en responder el test de cancelación de la A presentan una disminución en el promedio académico de -0.0043 (-0.0095; 0.0007 ). También se encontró que por cada aumento en la prueba de lectura crítica en Saber 11 , el promedio académico aumenta en 0.0094 (-0.0003; 0.0192).

Figura 1. Matriz de dispersión de los valores del rendimiento académico, la prueba lectura crítica en saber 11 y el desempeño en tareas de atención y memoria.

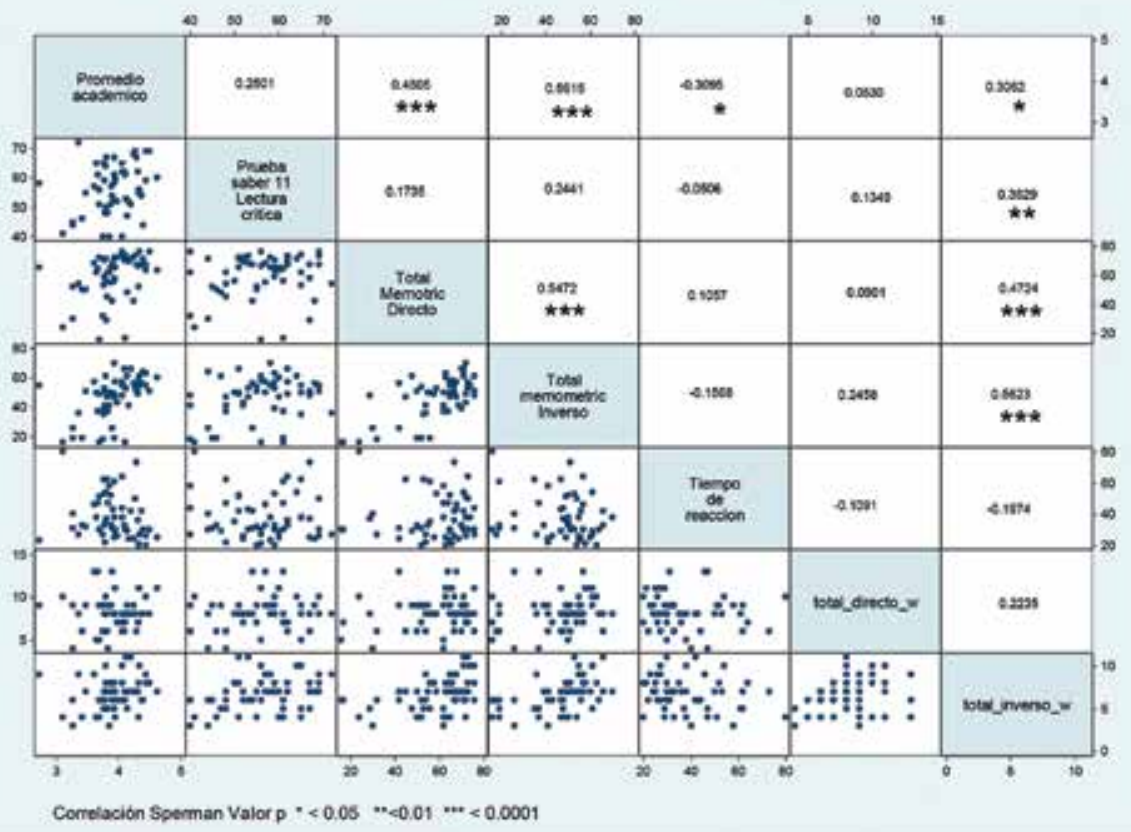


Tabla 2. Análisis bivariado y múltiple

\begin{tabular}{ccccc}
\hline Variable & B crudo (IC 95\%) & Valor p & B ajustado (IC 95\%) & Valor p \\
\hline $\begin{array}{c}\text { Total memometric } \\
\text { inverso }\end{array}$ & $0.0138(0.0087 ; 0.0190)$ & 0.000 & $0.0111(0.0058 ; 0.0163)$ & 0.000 \\
\hline $\begin{array}{c}\text { Tiempo de reacción test } \\
\text { de cancelación de la A }\end{array}$ & $-0.0073(-0.0136 ;-0.0009)$ & 0.024 & $-0.0043(-0.0095 ; 0.0007)$ & 0.094 \\
\hline $\begin{array}{c}\text { Prueba lectura crítica } \\
\text { saber 11 }\end{array}$ & $0.0124(0.0020 ; 0.0229)$ & 0.020 & $0.0094(-0.0003 ; 0.0192)$ & 0.059 \\
\hline$R^{2}$ ajustado & 0.4807 & & & \\
\hline
\end{tabular}

Fuente: Elaboración propia.

\section{Conclusiones}

En la muestra con un 95\% de confiabilidad se pudo establecer mediante la correlación de Spearman que el puntaje obtenido por los participantes en directo e inverso memometric tiene una relación directamente proporcional con el desempeño académico. Lo anterior es similar a los hallazgos de otros estudios, donde la inteligencia, aparece en la investigación psicológica como el primer predictor del rendimiento académico, seguido por variables, como la atención, la memoria, la motivación, los procesos afectivos y personales. (Muelas Chamorro-Premuzic \& Beltrán Furnham, 200611); Chamorro-Premuzic, Quiroga \& Colom, 2009; Colom \& Flores-Mendoza, 2007; Deary, Strand, Smith \& Fernández, 2007; Jensen, 1981; McGrew \& Knopik, 1993; Novak, Tsushima \& Tsushima, 1991; Pérez, González y Beltrán, 2009; Sternberg, Grigorenko \& Bundy, 2001; Vygotsky, 1978).

De acuerdo con el objetivo de este estudio, merece señalarse la relación hallada entre la memoria de trabajo evaluada por memometric en orden inverso y el rendimiento académico. No obstante, teniendo en cuenta la multicausalidad (motivación, emoción, relaciones de poder) de la variable, no es posible considerarla como predictor.
Barreyro, Cevasco, Burin, \& Molinari (2012), Bohn-Gettler \& Kendeou (2014), Currie \& Cain (2015), Prat et al.(2016), Van Dyke, Johns, \& Kukona (2014), Wang \& Gathercole (2013) han demostrado la vinculación de la memoria de trabajo con la comprensión de textos, empleando modelos de ecuaciones estructurales (McVay \& Kane, 2012) al igual que su relación con la capacidad de sostener la atención (Baddeley, 2010, 2012; Cowan, 2012). Tal como lo afirma Bohn-Gettler \& Kendeou (2014) y Prat, Seo, \& Yamasaki (2016) la memoria de trabajo ocupa un papel central en la comprensión del texto y en la generación de inferencias, porque permite el almacenamiento e integración de las representaciones.

Los hallazgos de la investigación son coherentes con las afirmaciones de Berninger y Richards (2002) quienes sostienen que a pesar de las relaciones halladas entre rendimiento académico y los componentes de la memoria a corto plazo y de trabajo, estas relaciones pueden no estar presentes en todos los casos. De igual manera, teniendo en cuenta que el rendimiento académico es un resultado final multifactorial, no es posible inferir que una buena o mala memoria de trabajo se reflejará en un alto o bajo rendimiento 
académico (Zapata, Los Reyes, Lewis y Barceló, 2009).

Por otro lado, los resultados en la prueba de lectura crítica Saber 11 presentan correlación con la subprueba de retención de dígitos total inverso de Wechsler, lo anterior puede explicarse por la implicación que tiene la memoria y atención en los procesos de comprensión y significado en la decodificación y análisis sintáctico de los textos (Abusamra y Joanette, 2012). Sin embargo,a pesar de hallarse una relación, el valor predictivo es débil, resultados similares a los encontrados por Pereira, Hernández y Gómez (2011).

Según los hallazgos, es posible inferir que la prueba Memometric es una herra-mienta que permite la evaluación de la memoria operacional a través de la tarea de retención de dígitos directos por medio visual. Sin embargo, se recomienda hacer estudios que permitan determinar la validez del instrumento.

En conclusión, el rendimiento académico y la memoria de trabajo constituyen variables complejas de estudiar, debido al carácter multifactorial. Se recomienda para próximas investigaciones evaluar los recursos afectivos del estudiante y los ambientes de aprendizaje.

\section{Referencias}

Abusamra, V., Yves. J. (2012). Editorial. Lectura, escritura y comprensión de textos: aspectos cognitivos de una habilidad cultural. Revista Colombiana de Psiquiatría, 37(1)

Albarracín, A., Dallos, I., Conde, C. (2008). Implementación de una prueba automatizada para la evaluación de memoria operacional: Memonum. Revista Colombiana de Psiquiatría, 37, 169-181.

Álvarez, L., González, P., Núñez, J., González, J., Álvarez., D. Bernardo, A. (2007). Programa de intervención multimodal para la mejora de los déficit de atención. Psicothema, 19(4), 591-596.

Ardila, A., Rosselli, M., Puente, A. E. (1994). Neuropsychological evaluation of the Spanish speaker. New York: Plenum Press.
Baddeley, A. (1992). Working Memory. Science, 255(5044), 556-559. https://science.sciencemag. org/content/255/5044/556

Baeten, M., Kyndt, E., Struyven, K., \& Dochy, F. (2010). Using student-centred learning environments to stimulate deep approaches to learning: Factors encouraging or discouraging their effectiveness. Educational Research Review, 5(3), 243-260.

Barreyro, J. P., Injoque-Ricle, I., Formoso, J., \& Burin, D. I. (2017). El rol de la memoria de trabajo y la atención sostenida en la generación de inferencias explicativas. Liberabit, 23(2), 235-247.

Berninger, V. W., Richards, T. (2002). Brain literacy for educators and psychologists. Academic Press.

Cáceres, M. T., y Salas, M. (2017). Resultados del rendimiento académico de las materias lingüísticas en secundaria obligatoria. Estudio de caso de un centro educativo público en España (2001-2014). RAEL: Revista Electronica de Lingüística Aplicada, 16(1) 57-69.

Castillo, A., Marín, A. (2006). Redes atencionales y sistema visual selectivo. Universitas Psychologica, 5(2), 305326. http://www.scielo.org.co/scielo.php?script=sci arttext\&pid $=$ S1657-92672006000200009\&lng $=$ en $\bar{\varepsilon}$ tlng $=$ es.

Chamorro-Premuzic, T., \& Furnham., A. (2008). Personality, intelligence and approaches to learning as predictors of academic performance. Personality and individual differences, 44(7), 1596-1603.

Chamorro-Premuzic, T., Quiroga, M., A., \& Colom, R. (2009). Intellectual competence and academic performance: A Spanish study. Learning and individual differences, 19(4), 486-491.

Cooley, E.L., Morris, R.D. (1990). Attention in children: A neuropsychological based model for assessment. Dev Neuropsychol, 6, 239-74.

Colmenero, J., Catena, A., Fuentes, L. (2001). Atención visual: Una revisión sobre las redes atencionales del cerebro. Anales de psicología, 17(1), 45-67.

Colom, R., \& Flores-Mendoza, C. . E. (2007). Intelligence predicts scholastic achievement irrespective of SES factors: Evidence from Brazil. Intelligence, 35(3), 243-251.

Deary, I., . J., Strand, S., Smith, P., \& Fernandes, C. (2007). Intelligence and educational achievement. Intelligence, 35(1), 13-21.

Fernández, D. (2008). Anatomía-funcional de la atención. http://www18.homepage.villanova.edu/diego. fernandezduque/Publications/capitulo15 atencion. pdf

González, B., Vega, V. (2010). Prácticas de lectura y escritura en cinco asignaturas de diferentes programas de la Universidad Sergio Arboleda. Civilizar: Ciencias Sociales y Humanas, 10(18), 101-116.

Lotti, M., Salim, R., Raya, F., y Dori, M.G. (2008). Una experiencia de formación docente sobre lectura comprensiva de textos científicos. Revista Iberoamericana de Educación, 45(3) 1-5. 
Muelas, Á., y Beltrán, J. A. (2011). Variables influyentes en el rendimiento académico de los estudiantes. Revista de Psicologéa de la Educación (6), 173-195.

Prada, E., Pineda, G., Mejía, M., Conde, C. (2010). Prueba computarizada Memonum: efecto de intervalos y distractores sobre la memoria de trabajo en mujeres mayores de 50 años. Universitas Psychologica, 9(3), 893-906.

Pereira, C., Hernández, G., Gómez, I. (2011). El valor predictivo de los exámenes de Estado frente al rendimiento académico universitario. Educación y educadores, 14(1) 51-65.

Puente, A. (2010). Prácticas de cognición y aprendizaje: fundamentos psicológicos. Ediciones Pirámide.
Tirapu, J., García, A., Rios, M., Pelegrín, C. (2011). Funciones ejecutivas. Rehabilitación neuropsicológica intervención y práctica clínica. Dialnet. https://dialnet. unirioja.es/servlet/libro?codigo $=685524$

Vázquez, M., Vaquero, E., Cardoso, M., Gómez, C. (2001). Atención basada en el espacio versus atención basada en el objeto: Un estudio psicofisiológico. La atención: un enfoque pluridisciplinar (2) 91-102. https://dialnet.unirioja.es/servlet/ articulo? codigo $=5105169$

Zapata, L. F., Los Reyes, C. de., Lewis, S., y Barceló, E. (2009). Memoria de trabajo y rendimiento académico en estudiantes de primer semestre de una universidad de la ciudad de Barranquilla. Psicología desde el Caribe (23), 66-82. 